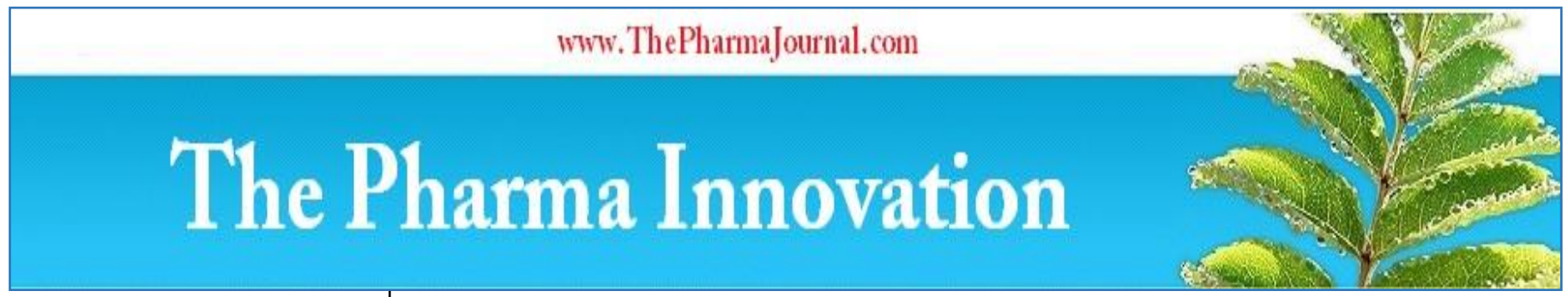

ISSN (E): 2277 - 7695

ISSN (P): 2349-8242

NAAS Rating: $\mathbf{5 . 0 3}$

TPI 2020; SP-9(12): 19-25

(C) 2020 TPI

www.thepharmajournal.com

Received: 16-10-2020

Accepted: 19-11-2020

Jagan Mohan Reddy K

Assistant Professor, Department

of Veterinary Surgery and

Radiology, College of Veterinary

Science, Rajendranagar,

Hyderabad, Telangana, India

\section{Clinical study on the use of supracondylar femur plate (J plate/ hockey stick plate) for repair of supracondylar femur fractures in cats}

\section{Jagan Mohan Reddy K}

DOI: https://doi.org/10.22271/tpi.2020.v9.i12Sa.5393

\begin{abstract}
The current study was undertaken to evaluate the clinical efficacy of Supracondylar femur plate for repair of supracondylar femur fractures in cats. Out of the clinical cases presented, $1 \%$ of cases in cats correspond to fractures, in which $25 \%$ correspond to supracondylar femur fractures. This study was conducted on six cats with age ranging from 4-11months, in 5 female and 1 male cat presented to the Department of Veterinary Surgery and Radiology at the College of Veterinary Science, Hyderabad with the chief complaint of falling from an height. These six cases of fractures were diagnosed by clinical signs, orthopaedic examination and survey radiography. The symptoms observed in the cats presented for treatment were pain on manipulation, abnormal angulation and lameness immediately after trauma, swelling, non-weight bearing, dangling of the limb and crepitation at the fracture site. Pre-operative radiographic examination in plain orthogonal views, i.e., cranio-caudal and medio-lateral radiographs revealed supracondylar fractures in all cats. These fractures were stabilized with $2.0 \mathrm{~mm}$ supracondylar femur plate 1 in right femur and 5 in left femur. Good implant stability throughout the treatment period without any complications was achieved all cats. In all six cats in the present study showed partial weight bearing on the $1^{\text {st }}$ post- operative day. All cats showed normal weight bearing at rest, the weight bearing of the affected limb while walking from $2^{\text {nd }}$ Post-operative day. All cats achieved complete weight bearing by $15^{\text {th }}$ post-operative day. i.e. from Grade V to Grade I. The radiographs obtained on $30^{\text {th }}$ day Post-operative day revealed proper apposition, decreased fracture gap, appearance of progressive bridging callus with adequate radio-density in all 6 cats, and on $60^{\text {th }}$ day revealed continuity of complete corticomedullary cavity and compete bone formation without any complications with fast healing. Based on present study, it was concluded that supracondylar femur plate was successful in the treatment of supracondylar femur fractures in cats.
\end{abstract}

Keywords: Supracondylar femur plate, internal fixation, suprcondylar femur fracture repair, cats.

\section{Introduction}

The incidence of femoral fractures account for $35 \%$ of pelvic limb fractures and $24 \%$ of all fractures in dogs and cats ${ }^{[1]}$. Fractures of the distal femur have been found to constitute 18.2 to 25.7 per cent of all femoral fractures in the dog and cat ${ }^{[2,3]}$. The incidence of distal femur fractures in dogs accounts for $37.13 \%$ as reported ${ }^{4}$. Most of these distal fractures occur in immature animals and involve the growth plate. Among 203 fractures in dogs and cats, it is reported that 59.1 per cent were with fractures of the distal femur. Supracondylar fractures are uncommon in dogs, and they account for $30 \%$ of fractures of the distal end of the femur but only $6 \%$ of all fractures of the femur ${ }^{[5]}$.

Surgical treatment is recommended for almost all supracondylar femoral fractures in the dog and cat. Because of limited bone stock available for fixation, particularly with comminuted or associated epiphyseal fractures, reduction and fixation of supracondylar fractures can be remarkably challenging. To address these challenges, numerous methods of stabilization have been described, including (1) bone plates, (2) interlocking nails, (3) Kirschner wires, (4) Rush pinning, (5) screw fixation in lag fashion, (6) intramedullary rods, and (7) external skeletal fixation ${ }^{[6]}$. The goal of fracture repair is to achieve complete bone healing with optimum function in as little time as possible. Modern fracture stabilization techniques and implants provide outstanding stability enabling a more rapid return to activity. The value of a rapid return to activity includes decreased muscle atrophy, decreased weight gain, maintenance of joint range of motion, minimization of decubital injuries, improved patient attitudes, and higher client satisfaction ${ }^{[7]}$. Various internal fixation devices have been used to repair the long bone fractures depending upon the stability required.
Corresponding Author: Jagan Mohan Reddy K Assistant Professor, Department of Veterinary Surgery and Radiology, College of Veterinary Science, Rajendranagar, Hyderabad, Telangana, India 
Nonlocking bone plates achieve fixation of the fracture by friction generated by the application of a well-contoured plate to the bone surface with screws. When applied properly, bone plates effectively resist the axial loading, bending, and

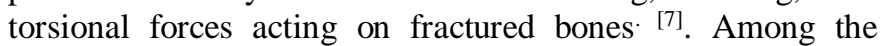
internal fixation techniques, bone plating was widely used because bone plates have the potential to restore rigid stability to the reconstructed fracture bone and also effectively resist the axial loading, bending and torsional forces acting on the fractures bones.

Plates specifically designed for the distal end of the femur, featuring a greater screw-hole density, may be used. These condylar plates are commonly referred to as "hockey stick plates" because of their characteristic appearance. Although these plates may substantially improve construct stability, they are only available in a few sizes ${ }^{[8]}$. Which is very useful for achieving a rigid plate fixation where there is limited bone for screw purchase due to the curvature of the femoral condyle in supracondylar fractures ${ }^{[9]}$.

\section{Materials and Methods Anamensis}

The cats presented for treatment were 3 Non-Descript and other 3 were Breed type and the mean age of the cats was $8.0 \pm 1.01$ months with ranging from 4 months to 11 months. The body weights of the cats ranged from 2-4 kg with a mean of $2 \mathrm{~kg}$. The cause of fractures in this group was falling from a height. The fractures occurred in 1 male cat and 5 female cats. The mean time of gap between the time of fracture and treatment was 5 days with a range of 3- 7 days.

\section{Pre-Operative Observations}

\section{Pre-operative Clinical Observations}

The six cats presented for treatment supracondylar femur fractures exhibited symptoms like sudden onset of pain and lameness immediately after a trauma. There were symptoms like swelling, dangling of the limb, non-weight bearing and abnormal angulation of the limb at the fracture site. None of the cats had neurological deficit. All the six cats had closed fractures.

\section{Pre-operative Radiographic Observations}

Two plain orthogonal views of medio-lateral and craniocaudal radiographs of the affected limbs including the proximal and distal joints confirmed fractures ${ }^{[10]}$. Out of six cats, the radiographs revealed Supracondylar fracture of femur all fractures are closed, one in right hindlimb and five in left hindlimb. Pre-operative radiographs of the cats with femur fractures were presented in fig. 1 to 6 .
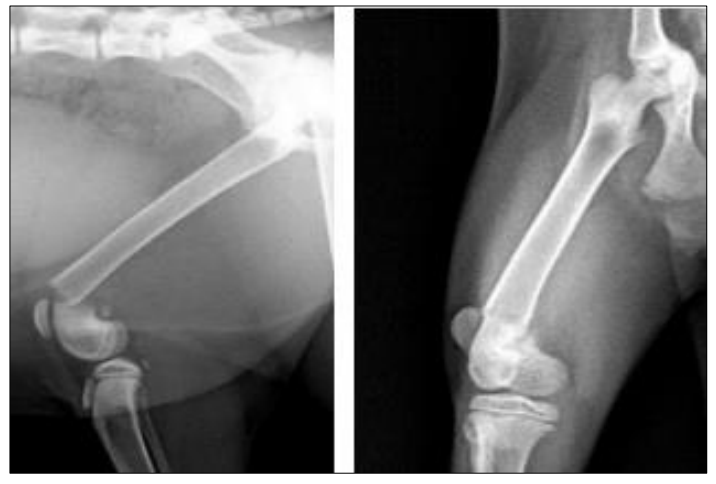

Fig 1: Skiagram showing supracondylar fracture of femur in a cat (medio-lateral and Cranio-caudal view)- Case 1, Pre-operative day

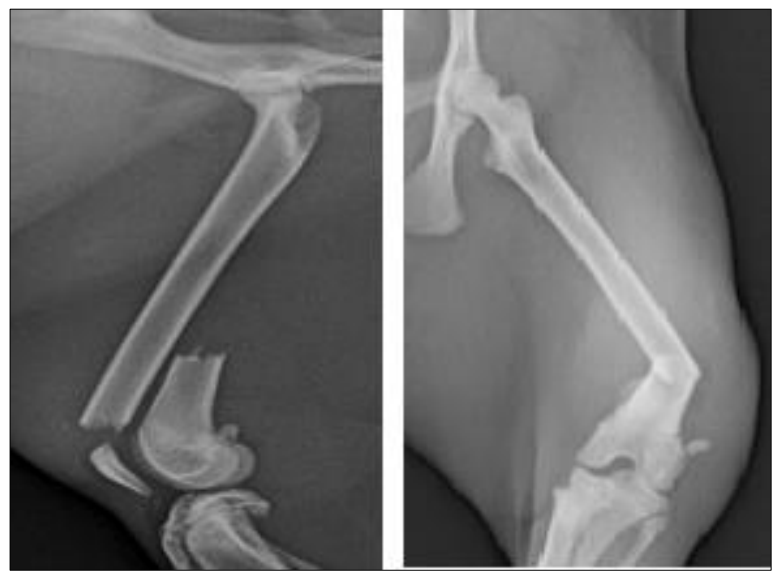

Fig 2: Skiagram showing supracondylar fracture of femur in a cat (medio-lateral and Cranio-caudal View) - Case 2 Pre-operative day
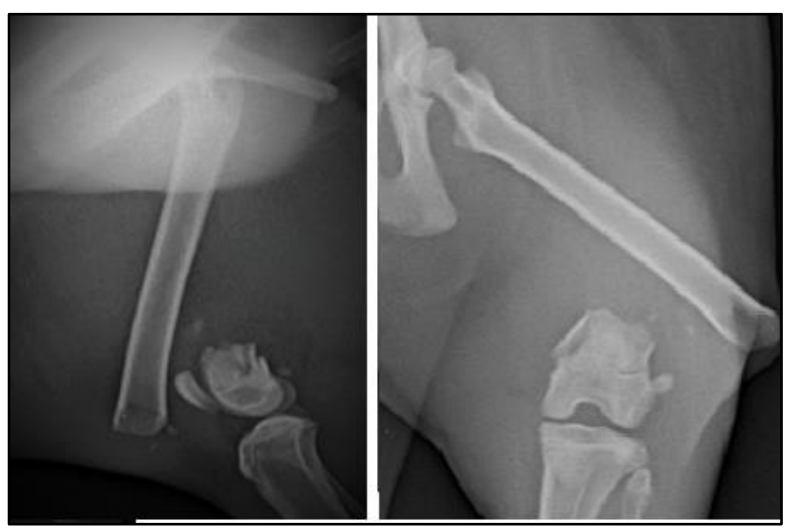

Fig 3: Skiagram showing supracondylar fracture of femur in a cat (medio-lateral and Cranio-caudal view)-Case 3, Pre-operative day

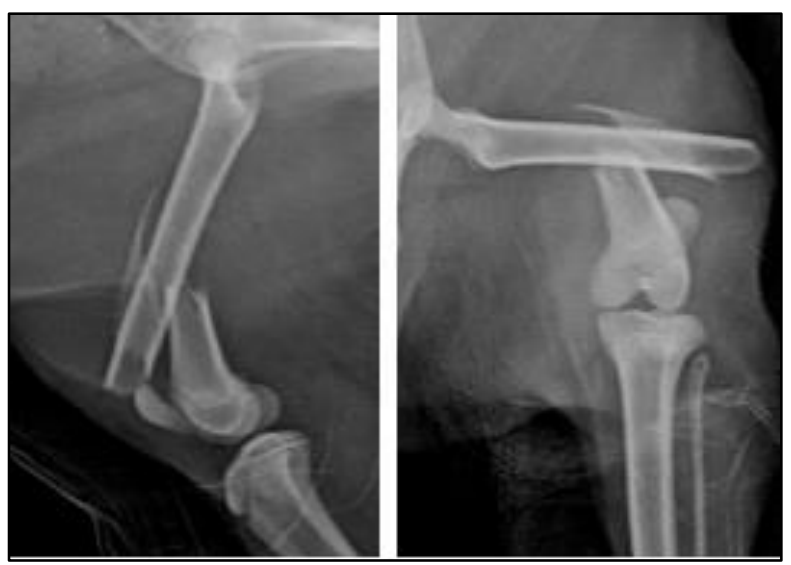

Fig 4: Skiagram showing supracondylar fracture of femur in a cat (medio-lateral and Cranio-caudal view)- Case 4, Pre-operative day

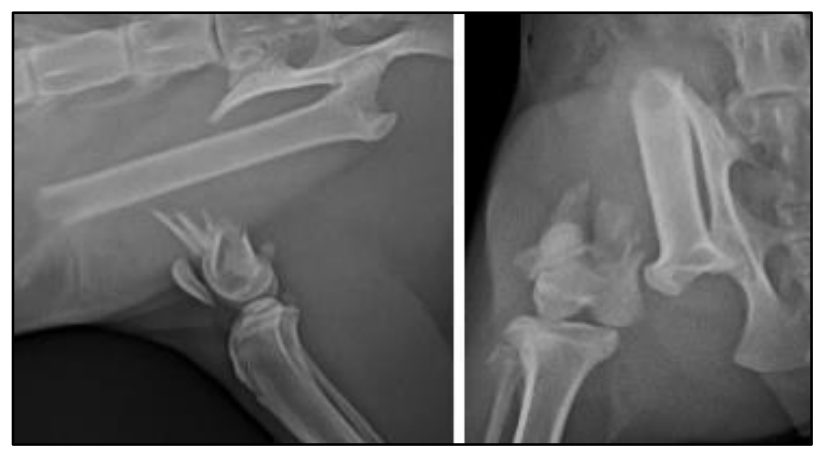

Fig 5: Skiagram showing supracondylar fracture of femur in a cat (medio-lateral and Cranio-caudal view) - Case 5, Pre-operative day 


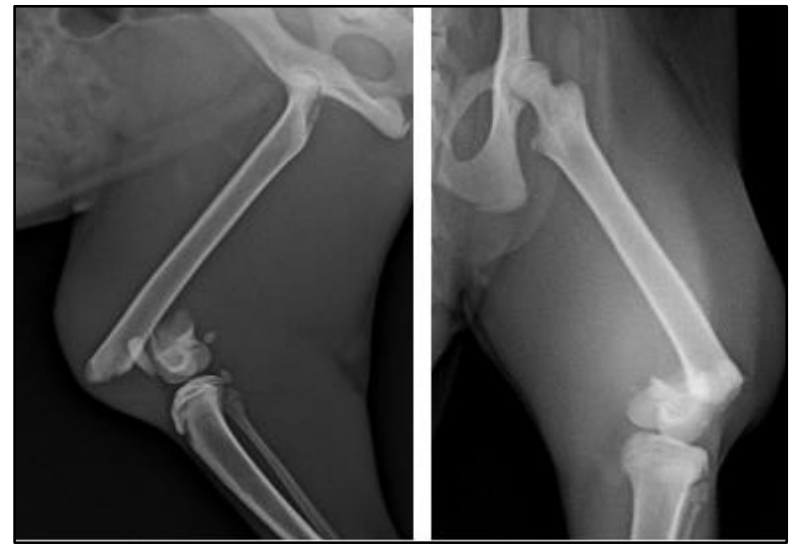

Fig 6: Skiagram showing supracondylar fracture of femur in a cat (medio-lateral and Cranio-caudal View) - Case 6, Pre-operative day

\section{Planning of Surgery}

Measurements obtained from the pre-operative radiographs of the affected limb like length of the bone and transcortical diameter at different regions proved vital in selecting the length of the supracondylar plates and the length of the screws to be used precisely in fracture fixation of femur.

\section{Patient preparation}

The affected limb was aseptically prepared by clipping the hair from a wide area surrounding the fracture site taking care to include upper and lower joints. The operative site was shaved and scrubbed using povidone-iodine surgical scrub, followed by the application of surgical spirit. Similarly, the skin was also prepared over the cephalic vein on both fore limbs for intravenous injections. Normal saline was infused intravenously throughout the duration of surgery.

\begin{abstract}
Anaesthesia
Atropine sulphate at the rate of $0.04 \mathrm{mg} / \mathrm{kg}$ body weight was administered subcutaneously as pre-anesthetic medication followed 10-15 minutes later by xylazine hydrochloride at the rate of $1 \mathrm{mg} / \mathrm{kg}$ body weight intramuscularly ${ }^{[11]}$. Ten minutes later, general anaesthesia was induced with intramuscular injection of ketamine hydrochloride at the rate of $10 \mathrm{mg} / \mathrm{kg}$ body weight ${ }^{[12]}$. Following induction, the cats were intubated with endotracheal tubes of suitable size. Anaesthesia was maintained with intravenous injection of propofol at the rate of $4 \mathrm{mg} / \mathrm{kg}$ body weight. Additional doses of propofol were also administered whenever necessary during surgical procedure through the intravenous line.
\end{abstract}

\section{Positioning of the Animal}

The cats with supracondylar fracture of femur were positioned in lateral recumbency with the fractured limb up.

\section{Materials Used \\ Orthopaedic Instruments}

A general surgical instrument set and orthopaedic instruments as needed for a particular procedure were used. Orthopaedic instruments including Gelpi retractors, Bone holding forceps, Hohmann's retractors, Senn retractors, Orthopaedic hexagonal screw drivers, $1.5 \mathrm{~mm}$ Drill bit, Depth gauge and Low speed high torque electric drill were used for performing Supracondylar Plating.

\section{Implants}

Supracondylar femur plate $2.0 \mathrm{~mm}$ thickness were used in six cats. Selection of appropriate length was determined by the weight of the cat and pre-operative measurements in mediolateral view for thickness and length of the plate by measuring the length and width of the femur. In the present study, used $2.0 \mathrm{~mm}$ thickness left supracondylar plate in 5 cats and 2.0 $\mathrm{mm}$ thickness right supracondylar plate in one cat long with $1.5 \mathrm{~mm}$ thickness K-wire for additional support. The results indicated that the use of 7 holed $2.0 \mathrm{~mm}$ supracondylar plate and self-tapping $2.0 \mathrm{~mm}$ cortical screws varying from $10 \mathrm{~mm}$ to $18 \mathrm{~mm}$ in length were used in six cats were found adequate without any complications.

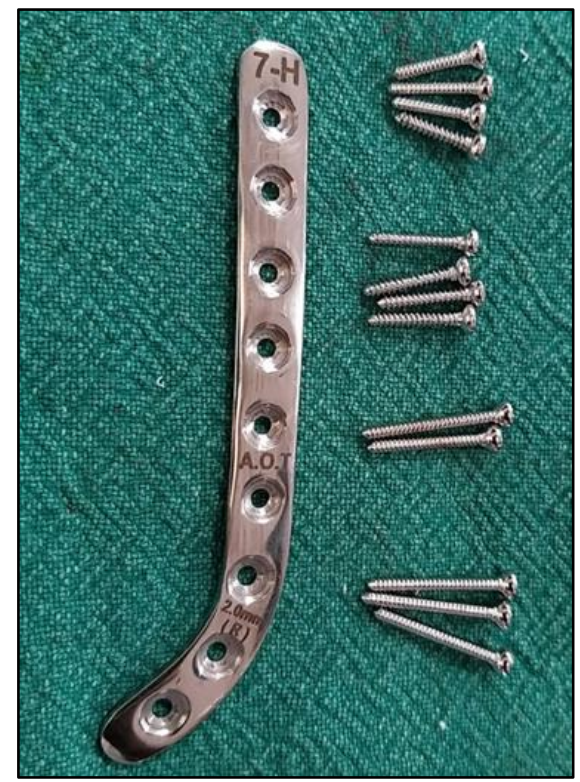

Fig 7: $2.0 \mathrm{~mm}$ Supracondylar femur plates self-tapping cortical screws used in cats

\section{Surgical Procedure}

The cranio-lateral border of thigh approach was used to expose the distal femur and stifle Joint through a lateral incision for repair of supracondylar fracture of femur bone to perform supracondylar femur plating technique in cats [13] (Fig.8)

Following the surgical exposure of the fracture site (Fig.9) as described, the fracture fragments were aligned and reduced to restore the length and correct rotational orientation (Fig. 10). Supracondylar plate was then placed over the bone and the plate was held in position with bone holding forceps. It was ensured that, the alignment was correct before securing the plate to the bone with cortical screws. The bone was drilled with $1.5 \mathrm{~mm}$ drill bit which corresponded with the inner core diameter of the screw using a low speed high torque electric drill. The length of the screws needed for application of supracondylar femur plate in each patient was determined by measuring the transcortical diameter of femur different distances from the fracture site of respective bones from the pre-operative radiographs and was confirmed during the surgical procedure by using a depth gauge. A screw of suitable length was then placed at the drilled hole and tightened using a hexagonal orthopaedic screw driver until the taper end of screw exited the far cortex to secure the supracondylar femur plate to the bone. Bone plating was accomplished by insertion of additional screws in both proximal and distal fracture fragments leaving the fracture line (Fig.11) 


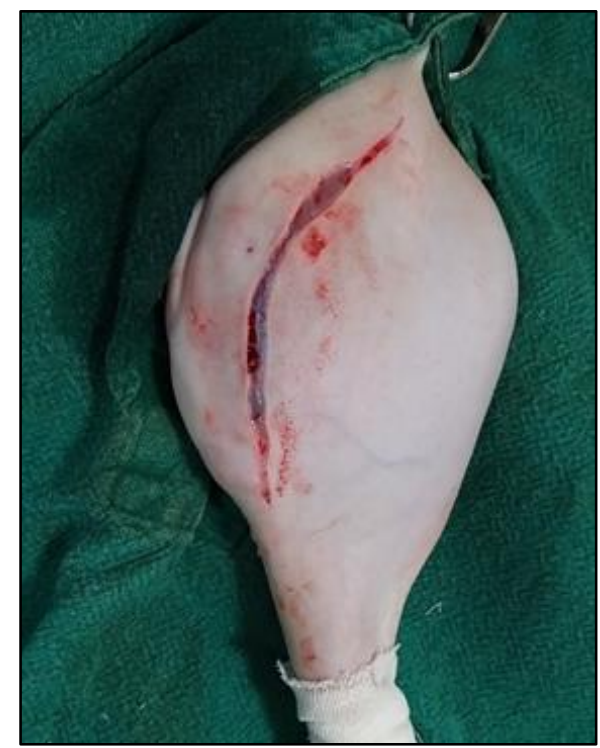

Fig 8: Skin incision made from trochanter major to lateral condyle of femur

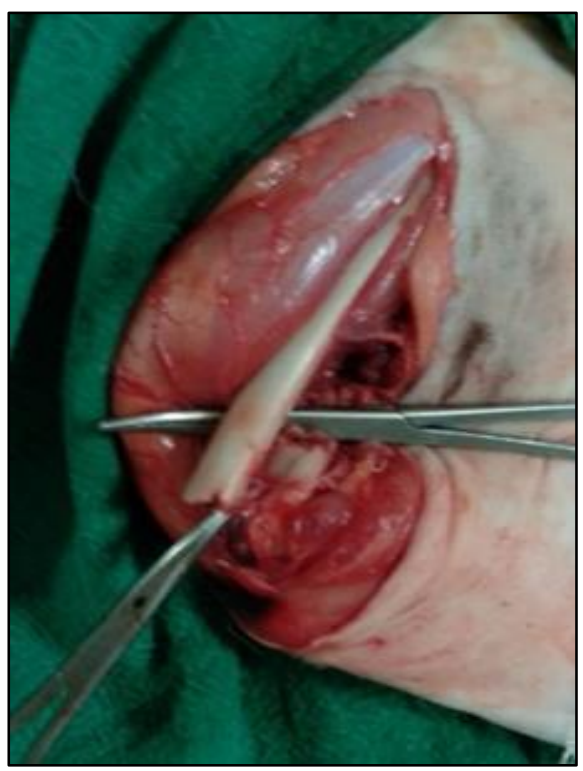

Fig 9: Femoral fractured fragments were exposed

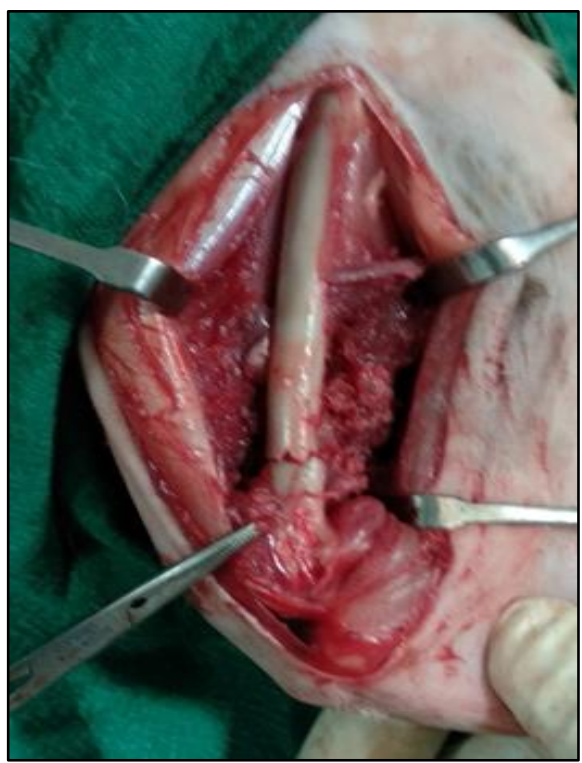

Fig 10: Fracture fragments were aligned and reduced to restore the length and correct rotational orientation

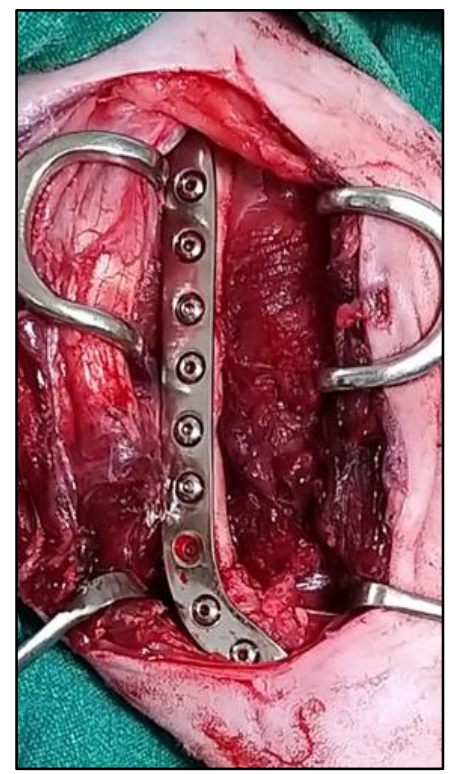

Fig 11: $2.0 \mathrm{~mm}$ Suparcondylar femur plate used in this group of cats in place

\section{Closure of the incision}

In case of femoral approach, the fascia lata was sutured with 2-0 polyglactin 910 in a simple continuous suture pattern. Subcuticular sutures were applied with 2-0 polyglactin 910 . Skin incision was closed with a row of cruciate mattress sutures of 2-0 polyamide (Fig.12)

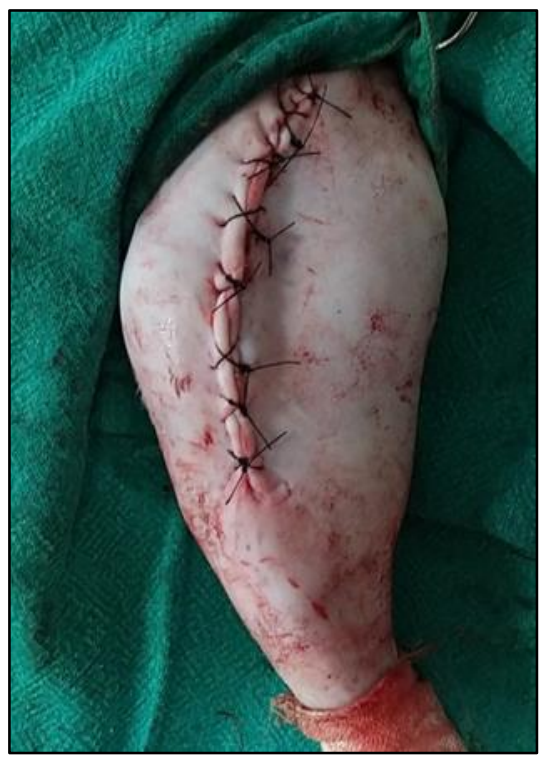

Fig 12: The skin incision was closed in a row of cruciate mattress sutures of 2-0 Polyamide

\section{Post-Operative Care and Management}

The suture line was covered with a thin layer of sterile gauze bandage dipped in 5\% povidone iodine solution. Over this, a thick layer of cotton pad was wrapped. It was then covered with gauze bandage and finally, a layer of surgical paper tape was applied. The dressing was replaced on every alternate day until the sutures were removed on the 12th post-operative day. Injection ceftriaxone sodium was administered at the rate of $25 \mathrm{mg} / \mathrm{kg}$ body weight as intramuscular injection for 7 days post-operatively. Injection meloxicam was administered once a day at the rate of $0.3 \mathrm{mg} / \mathrm{kg}$ body weight by intramuscular injection for 3 days post-operatively. Owners were advised to restrict the movement of the animal for the first 2 weeks of 
surgery and then to allow leash walking for the next few weeks.

\section{Results}

Clinical evaluation was carried out every alternate day to check for the presence of swelling, exudation and weight bearing in all the cats. The appearance of suture line was also examined every alternate day until the sutures were removed. The post- operative day on which the cat started bearing weight was recorded and graded. After suture removal, the cats were examined once in a week for the limb stability until fracture healing was considered satisfactory.

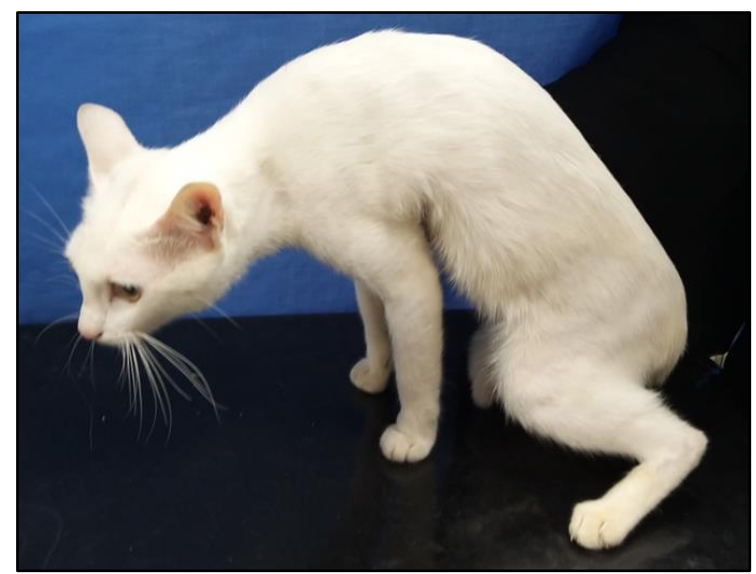

Fig 13: Showing complete weight bearing of affected hind limb on $15^{\text {th }}$ post-operative day

\section{Post-Operative Clinical Observations}

Dressing the surgical wound with $5 \%$ povidone iodine pads was found to be effective in keeping the site clean in all the cats. Application of Robert-Jones bandage provided satisfactory immobilization of the limb. The use of injection ceftriaxone sodium effectively prevented post-operative infection. The skin sutures were removed between $10^{\text {th }}$ to $12^{\text {th }}$ post-operative days in all the cats. None of the cats developed post-operative swelling and suture dehiscence and the surgical wounds healed well in all the cats without any complications.

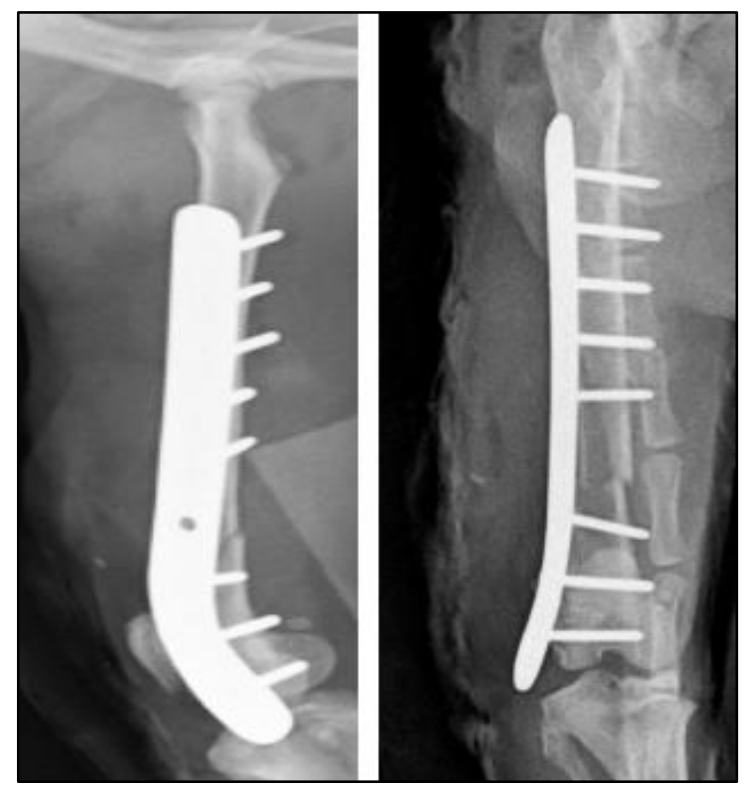

Fig 14: Skiagram showing apposition of left femur supracondylar fracture fragments (Medio-lateral and Cranio-caudal view) Case 2, Immediate post-operative day

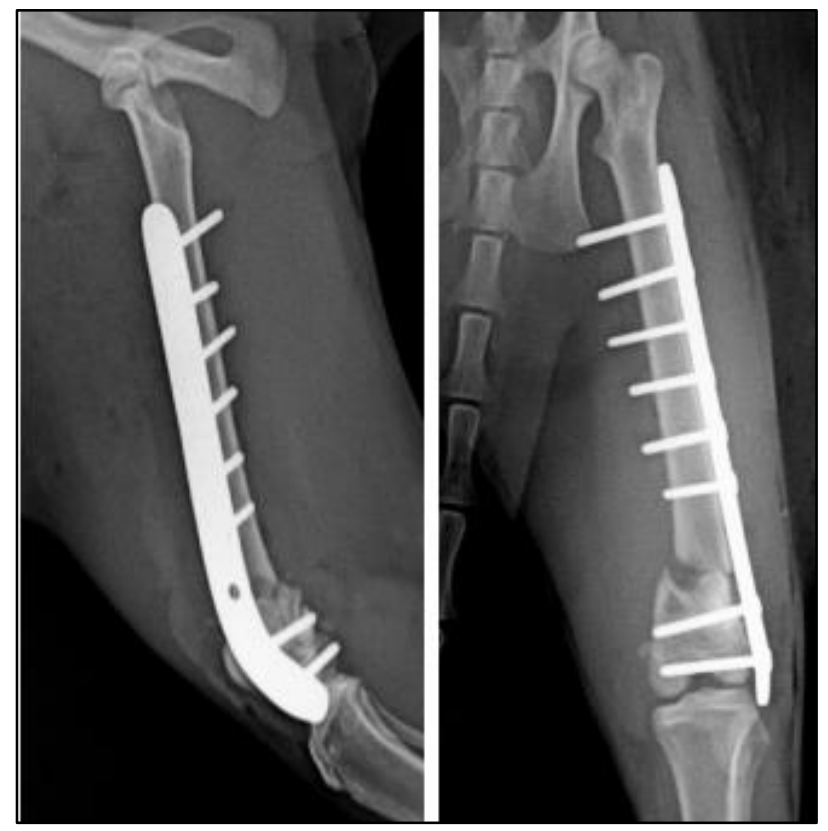

Fig 15: Skiagram showing apposition of left femur supracondylar fracture fragments (Medio-lateral and Cranio-caudal view) Case 3, Immediate post-operative day

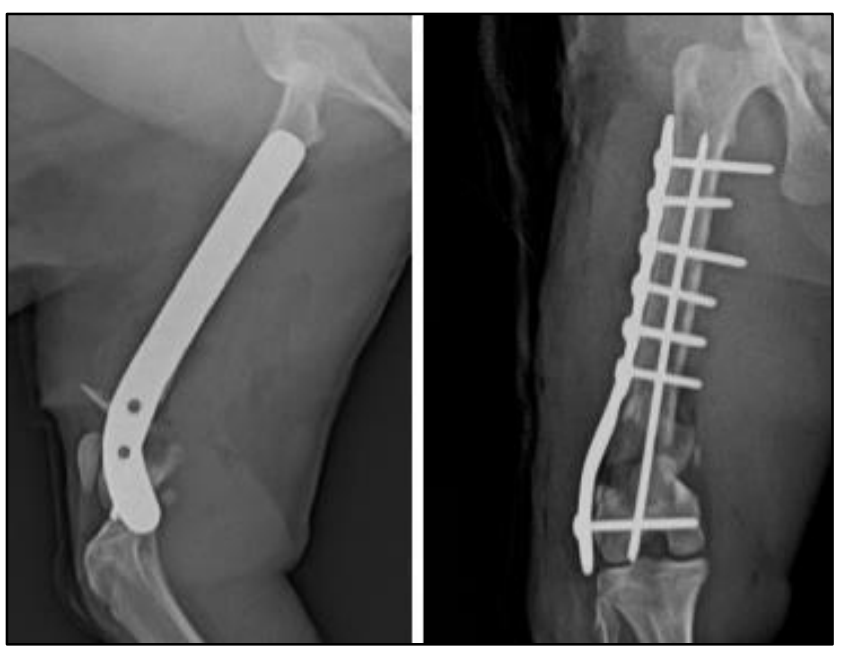

Fig 16: Skiagram showing apposition of Right femur supracondylar fracture fragments (Medio-lateral and Cranio-caudal view ) Group II- Case 3, Immediate post-operative day
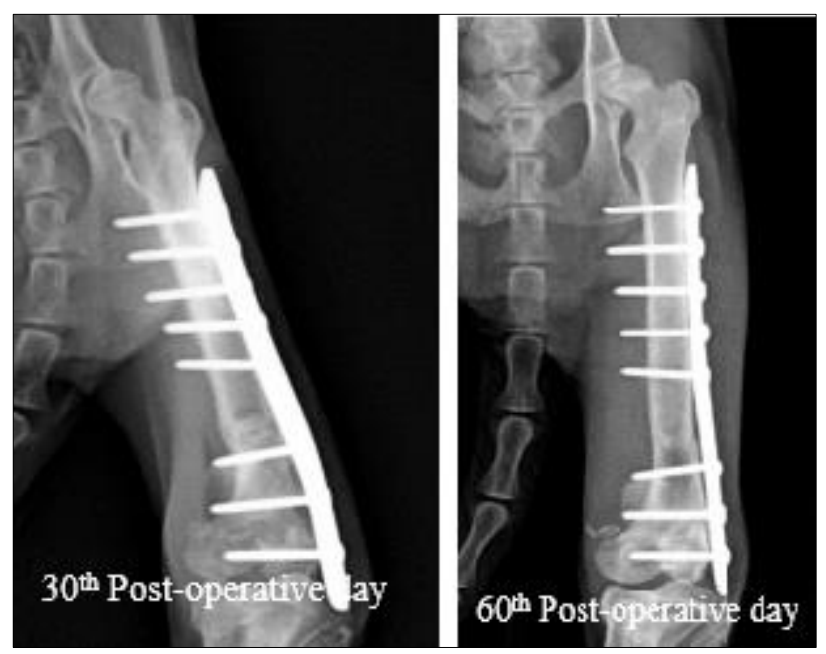

Fig 17: Skiagram showing progressive radiographic fracture healing of supracondylar left femur fracture in a cat (case. 2) 


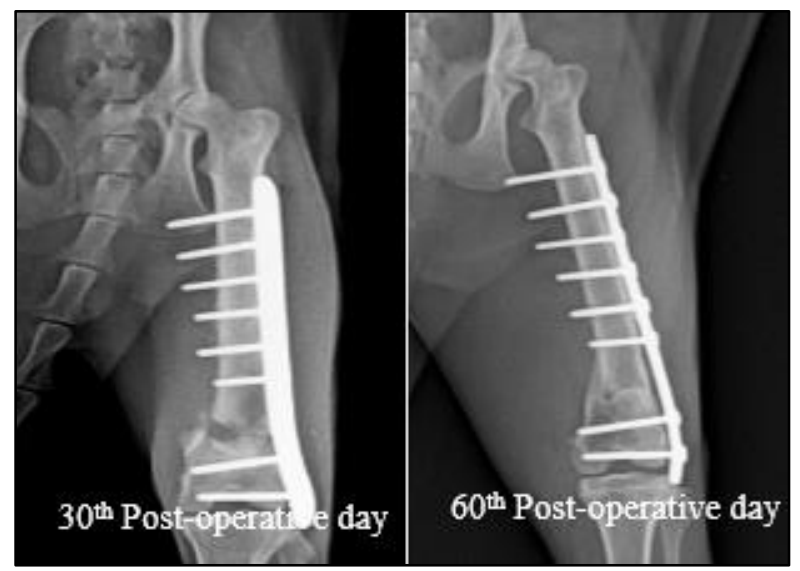

Fig 18: Skiagram showing progressive radiographic fracture healing of supracondylar left femur fracture in a cat (case. 3)

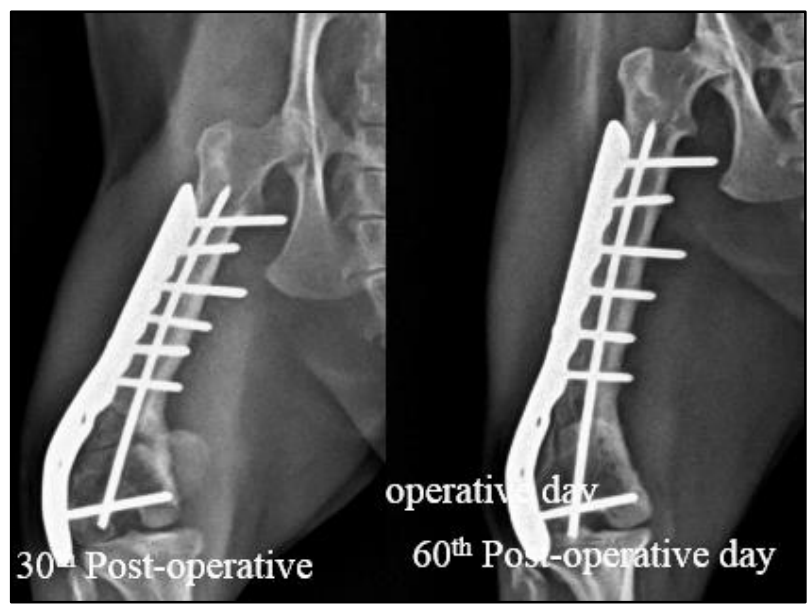

Fig 19: Skiagram showing progressive radiographic fracture healing of supracondylar Right femur fracture in a cat (case. 5)

\section{Lameness grading}

In all six cats in the present study showed partial weight bearing on the $1^{\text {st }}$ post- operative day. All six cats showed normal weight bearing at rest, the weight bearing of the affected limb while walking from $3^{\text {rd }}$ Post-operative day in one cat, $5^{\text {th }}$ Post-operative day in two cats, and $8^{\text {th }}$ Postoperative day in two cats and $15^{\text {th }}$ Post-operative day in one cat. (Fig.13). All six cats achieved complete weight bearing from grade $\mathrm{V}$ to grade $\mathrm{I}$ on $15^{\text {th }}$ post-operative day.

\section{Post-Operative Radiographic Observations}

Immediate Post-operative radiographic evaluation confirmed proper placement of supracondylar femur plate with selftapping cortical screws, and good apposition of the fractures fragments in all the six cats (Fig.No.14 to 16). Immobilization was considered satisfactory in all cases in this group. The radiographs obtained on $30^{\text {th }}$ day Post-operative day revealed proper apposition, decreased fracture gap, appearance of progressive bridging callus with adequate radio-density in all 6 cats. By $60^{\text {th }}$ post-operative day the fracture line disappeared and the callus became radio-dense with clear establishment of cortico-medullary canal in all 6 cats. The progressive radiographic fracture healing was showed in Fig. 17 to 19. In this group it is depicted good fracture healing on the radiographic evaluation ${ }^{[14]}$ in all cats without any complications.

\section{Discussion}

Lameness grading based on weight bearing was recorded in all cases pre-operatively, showed grade V lameness. Postoperatively, all 6 cats progressed to grade I lameness by the end of $15^{\text {th }}$ post-operative day and Post-operative radiographic evaluation confirmed proper placement of the supracondylar femur plate and screws, apposition and alignment of the fracture fragments in all the six cats ${ }^{[15]}$. Immobilization was considered satisfactory in all the cases. The plate length, size and position were appropriate in all the cases. Screw length, size and position were found to be appropriate in all the cases. Follow-up radiographs taken on $30^{\text {th }}$ post-operative days revealed primary bone healing with minimal callus formation. By $60^{\text {th }}$ post-operative day the fracture line disappeared and the callus became radio-dense with clear establishment of cortico-medullary canal in all 5 cats. Good implant stability throughout the treatment period without any complications was achieved in all cats and similar results was reported $[9,16,17]$.

\section{Conclusion}

Based on present study, it was concluded that Supracondylar femur plate was successful for the repair of femur fractures in cats with supracondylar femur fractures and offered good recompense and remarkable improvement in the limb function, with good fracture stability till the completion of the bone healing in all six cats. The application of supracondylar femur plate (J plate/ hockey stick plate) was found to be effective very for stabilization of supracondylar femur fractures in cats. In all six cats had no complications, and no owner insisted for removal of the implant.

\section{Acknowledgements}

The author is thankful to the cat owners for getting their cats for regular review for post-operative assessment of the supracondylar femur fracture cases.

\section{References}

1. Johnson AL, Smith CW, Schaeffer DJ. Fragment reconstruction and bone plate fixation versus bridging plate fixation for treating highly comminuted femoral fractures in dogs: 35 cases (1987-1997). J Am Vet Med Assoc 1998;213:1157-1161.

2. Stigen O. Femoral fractures in the dog and cat. I. Location. type of fracture and occurrence. Norsk Veteriner Tidsskrift 1986;98:13-18.

3. Stein H. Fractures of the femur of dogs and cats: analysis of case records from the Hannover Small Animal Clinic for 1985-1989.) Dissertation, Tierarztliche Hochschule Hannove 1990.

4. Harikrishna NVV. Plate osteosynthesis of distal femoral fractures using locking reconstruction plate, locking distal femoral head plate and locking 1-plates in dogs. Thesis submitted to TANVAS 2013.

5. Braden TD, Eicker SW, Abdinoor D, Prieur WD. Characteristics of 1000 femur fractures in the dog and cat. Vet. Comp. Orthop. Traumatol 1995;8:57-66

6. Lidbetter DA, Glyde MR. Supracondylar femoral fractures in adult animals. Compend Contin Educ Pract Vet 2000;22:1041-1055.

7. Hayashi K, Schulz KS, Fossum TW. Principles of Fracture Diagnoses and Management. In Textbook of Small Animal Surgery. Fossum T W, $5^{\text {th }}$ Ed Elsevier Health Sciences Missouri 2019,976-1025.

8. Guiot LP, Déjardin LM. Fractures of the Femur, In Veterinary surgery by Tobias, K.M., and Johnston, 
S.A.,Edn. $2^{\text {nd }}$. e -book-. Set. Elsevier Health Services 2018;2:1019-1070.

9. Meeson R. Making internal fixation work with limited bone stock. In practice 2017;39(3):98-106.

10. Guiot LP, Demianiuk RM, Dejardin LM. Fractures of the femur. In Veterinary Surgery Small Animal Vol.1 edited by Karen M. Tobias and Spencer A. Johnston. Edn. $1^{\text {st }}$ 2012;1:865-905.

11. Pardeshi GD, Ranganath L. Comparison of Type 1a and Type1b external skeletal fixation for tibial fracture repair in dogs. Indian Journal of Veterinary Surgery 2008;29(2):93-95.

12. Fattahian HR, Mohyeddin H, Molookpour H, Hoseinzadeh AR. Retrospective study of surgical treatment of various patellar luxations in dogs from 2004 to 2007. Iranian Journal of Veterinary Research 2011;12(1):56-60.

13. Johnson KA. Piermattei's Atlas of Surgical approaches to the bones and joints of the dog and cat $5^{\text {th }}$ edn. Elsevier 2014;374-379:388-391.

14. Cook JL, Tomlinson JL, Reed AL. Fluoroscopically guided closed reduction and internal fixation of fractures of the lateral portion of the humeral condyle: prospective clinical study of the technique and result in ten dogs. Vet. Surg 1999;28:315-321.

15. Piermattei DL, Flo GL, Brinker WO. Fracture classification, diagnosis, and treatment. Hand book of Small Animal Orthopedics and Fracture Repair, 5th Ed by Elsevier Missouri 2016, 24-149.

16. Liang B, Ding Z, Shen J, Zhai W, Kang L, Zhou L et al. A distal femoral supra-condylar plate: biomechanical comparison with condylar plate and first clinical application for treatment of supracondylar fracture International Orthopaedics (SICOT) 2012;36:1673-1679.

17. Smalle TM, Coetzee GL, Naude SH. Corrective wedge ostectomy for an atypical femoral procurvatum deformity stabilised with a supracondylar bone plate. Journal of the South African Veterinary Association 2018, 1019-9128. 